\title{
Identification of a biochemical link between energy intake and energy expenditure
}

\author{
Silvana Obici, Jiali Wang, Rahena Chowdury, Zhaohui Feng, Uma Siddhanta, \\ Kimyata Morgan, and Luciano Rossetti
}

Diabetes Research and Training Center, Albert Einstein College of Medicine, Bronx, New York, USA

Address correspondence to: Luciano Rossetti, Albert Einstein College of Medicine,

1300 Morris Park Avenue, Bronx, New York 10461, USA.

Phone: (718) 430-4118, or (719) 430-4215; Fax: (718) 430-8557; E-mail: rossetti@aecom.yu.edu.

Silvana Obici and Jiali Wang contributed equally to this work.

Received for publication February 12, 2002, and accepted in revised form April 11, 2002.

Obesity is the result of an imbalance between energy intake and energy expenditure. Using high-density DNA microarrays and Northern analyses, we demonstrated that the activation of a nutrient-sensing pathway, the hexosamine biosynthesis pathway (HBP), rapidly decreased the expression of a cluster of nuclear-encoded mitochondrial genes involved in skeletal muscle oxidative phosphorylation. Conversely, the expression of uncoupling protein-1 and of the same mitochondrial genes was increased in brown adipose tissue. Most important, these transcriptional changes were accompanied by a marked decrease in whole-body energy expenditure. Short-term overfeeding replicated this transcriptional pattern, suggesting that this adaptation to nutrient abundance occurs under physiological conditions. Thus, the activation of the HBP by nutrients represents a biochemical link between nutrient availability, mitochondrial proteins, and energy expenditure, and it is likely to play an important role in the regulation of energy balance.

J. Clin. Invest. 109:1599-1605 (2002). doi:10.1172/JCI200215258.

\section{Introduction}

The increasing prevalence of obesity in industrialized societies is largely due to excessive caloric intake. However, there are major variations in the extent to which an individual alters energy metabolism and gains weight in response to increased caloric intake $(1,2)$. Furthermore, base-line energy expenditure is a strong predictor of future weight gain (3). The physiological basis for individual variability in energy expenditure is still poorly understood. A sustained increase in food intake activates adipostatic responses, which tend to limit lipid storage by enhancing energy expenditure (4) and inhibiting appetite (5). However, consistent with Neel's "thrifty genotype" hypothesis, the increased availability of nutrients may also have biological effects designed to increase the efficiency of energy storage by limiting fuel oxidation and ATP production (6). Indeed, cells can monitor nutrient availability via the activation of nutrient-sensing pathways such as the hexosamine biosynthesis pathway (HBP) $(7,8)$. The final step in HBP is the formation of UDP- $N$-acetylglucosamine (UDP-GlcNAc), which is a product of intracellular glucose metabolism and is a main substrate for glycosylation of proteins. Many cytoplasmic and nuclear proteins are glycosylated on their serine and/or threonine residues by the addition of a single molecule of $O$-linked $\beta$ - $N$-acetylglucosamine (O-GlcNAc) (9). In particular, several transcriptional factors undergo this type of rapid modification, which causes changes in their activity and/or in their stability (Figure 1a) (10-13). A prominent role of HBP in the regulation of energy balance is suggested by its role in the modulation of leptin expression and insulin action in adipose tissue and skeletal muscle $(7,8,14-17)$.

\section{Methods}

Animals. Male Sprague-Dawley rats (Charles River Laboratories, Wilmington, Massachusetts, USA) were housed in individual cages and subjected to a standard 12-hour light-dark cycle. All rats received standard chow ad libitum (catalog no. 5001; Purina Mills Inc., St. Louis, Missouri, USA), with 59\% calories provided by carbohydrates, $20 \%$ by protein, and $21 \%$ by fat. Seven days prior to the in vivo studies, all rats underwent insertion of indwelling catheters into the right internal jugular vein and the left carotid artery as described previously (14). In the shortterm overfeeding experiments, rats were allowed to eat ad libitum a palatable high-fat diet (catalog no. 9389; Purina Mills Inc.) with $45 \%$ calories provided by carbohydrates, $33 \%$ by fat, and $22 \%$ by protein for 3 days prior to the clamp studies as previously described (18). Control rats received a daily allowance of palatable high-fat diet restricted at $80 \%$ of their pre-intervention caloric intake.

Clamp studies. All in vivo studies were performed in awake, unstressed rats fasted for 6 hours, using a euglycemic-hyperinsulinemic clamp technique, as described previously (14). GlcN (30 $\mu \mathrm{mol} / \mathrm{kg} / \mathrm{min}, n=11)$ or saline 
$(1.2 \mathrm{ml} / \mathrm{h})$ was infused for 4 hours. Plasma samples for the determination of plasma glucose were obtained at 10-minute intervals throughout the insulin infusion. Samples for the measurement of plasma insulin, leptin, and other plasma substrates were obtained at $0,60,120$, 180 , and 240 minutes. The total volume of blood sampled was about $5 \mathrm{ml}$ per animal; to prevent volume depletion and anemia, a solution $(1: 1 \mathrm{vol} / \mathrm{vol})$ of about $6 \mathrm{ml}$ of fresh blood (obtained by heart puncture from a littermate of the test animal) and heparinized saline (10 units $/ \mathrm{ml}$ ) was infused. At the end of the in vivo studies, rats were anesthetized (intravenously with $60 \mathrm{mg}$ of pentobarbital per $\mathrm{kg}$ ) and tissue samples were removed, quickly frozen in liquid nitrogen, and stored at $-80^{\circ} \mathrm{C}$ for subsequent analysis.

DNA microarray. Microarray slides were prepared by the Albert Einstein College of Medicine Microarray Facility (http://sequence.aecom.yu.edu/bioinf/funcgenomic. html and ref. 19). Approximately 9000 mouse cDNA clones, obtained from the I.M.A.G.E. Consortium (Integrated Molecular Analysis of Genomes and their Expression Consortium, Livermore, California, USA), were screened using hybridization protocols previously described (20). Analysis of microarray hybridizations was carried out with ScanAlyze software (ScanAlyze2, available at http://rana.lbl.gov/EisenSoftware.htm; Stanford University, Stanford, California, USA) initially created by Eisen and Brown (20). Equal amounts of cRNA from three samples were used for preparation of each probe. All data sets were normalized to total fluorescence, which represents the total amount of cRNA hybridized to a microarray. All data analyses were performed by using an average fold change, derived from three independent studies, of 1.7 or greater, since these changes could be detected and validated by Northern analysis.

Western blots. For O-GlcNAc analysis, rat skeletal muscle was pulverized in liquid nitrogen and homogenized in 4 volumes of ice-cold buffer $(10 \mathrm{mM}$ Tris- $\mathrm{HCl} \mathrm{pH}$ 7.9, $10 \mathrm{mM} \mathrm{MgCl} 2,0.5 \mathrm{mM}$ PMSF, $1 \mathrm{mg} / \mathrm{ml}$ each of aprotinin, leupeptin, pepstatin, and $5 \mathrm{mM}$ benzamidine). The homogenate was centrifuged at $2000 \mathrm{~g}$ for 10 minutes. Thirty micrograms of supernatant was loaded on a $10 \%$ SDS-PAGE and blotted on nitrocellulose filters. Immunoblots were performed with a mouse $\mathrm{mAb}$ specific for O-GlcNAc (Covance Research Products Inc., Richmond, California, USA).

Northern analyses. Results of microarray analysis for selected individual genes were verified using Northern analysis. RNA was isolated from muscle and adipose tissue with Trizol (GIBCO BRL; Life Technologies Inc., Rockville, Maryland, USA), and $20 \mu \mathrm{g}$ of total RNA was fractionated in denaturing gels and transferred to nylon membranes. DNA probes were labeled with ${ }^{32} \mathrm{P}$-dCTP using a random-priming technique (Stratagene, La Jolla, California, USA). After hybridization and high-stringency washing, membranes were exposed to radiographic films. The intensity of the autoradiographic bands was quantified by densitometry (ImageQuant software; Molecular
Dynamics, Sunnyvale, California, USA). $\beta$-Actin hybridization was used to normalize for RNA loading.

Calorimetry. Animals undergoing indirect calorimetry were acclimated to the respiratory chambers for 3-4 days before the clamp studies. Calorimetry was performed on 12 rats with indwelling catheters, randomized in two groups to receive either $\mathrm{GlcN}$ or saline infusion respectively, during a euglycemic hyperinsulinemic-clamp (see Clamp studies above). One day before the in vivo study, all rats were individually housed in the calorimeter cages, and data on base-line gas exchanges, activity, and food intake were collected. Immediately after the clamp studies, the rats were returned to the calorimeter for the postclamp monitoring of gas exchanges and activity, in the absence of food. Indirect calorimetry was performed with a computer-controlled open-circuit calorimetry system (Oxymax; Columbus Instruments Co., Columbus, Ohio, USA). Our instrument comprises four respiratory chambers with a stainless steel elevated wire floor. Each chamber is equipped with water bottle, food tray connected to a balance, and activity monitor. Oxygen consumption and carbon dioxide production were measured for each rat at 6-minute intervals. Outdoor air reference values were measured after every ten measurements. Gas sensors were calibrated daily with primary gas standards containing known concentrations of $\mathrm{O}_{2}, \mathrm{CO}_{2}$, and $\mathrm{N}_{2}$ (Tech Air, White Plains, New York, USA). A mass flow meter was used to measure and control air flow. Oxygen was measured by an electrochemical sensor based on limited-diffusion, metal-air battery. $\mathrm{CO}_{2}$ was measured with a spectrophotometric sensor. Respiratory quotient (RQ) was
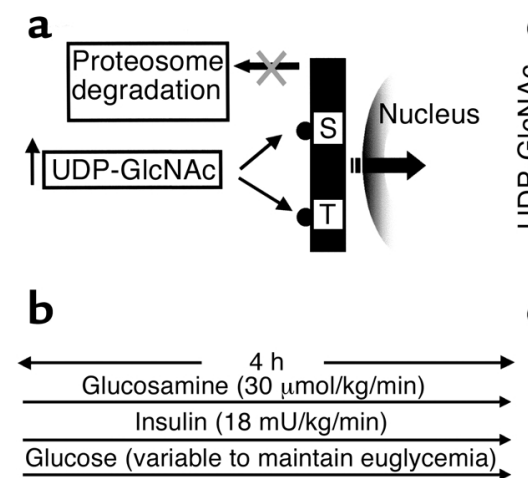

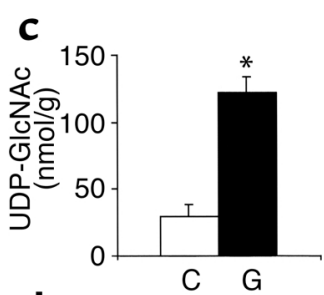

d

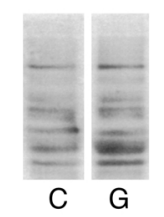

\section{Figure 1}

Activation of HBP increases O-linked glycosylation of nucleocytoplasmic proteins. (a) The enzyme OGTase links GlcNAc moieties to serine or threonine residues of transcriptional factors. O-GlcNAc induces conformational changes that render proteins refractory to proteosomal degradation and more available for modulation of transcription in the nucleus. (b) Schematic representation of experimental design. Acute infusion of glucosamine $(30 \mu \mathrm{mol} / \mathrm{kg} / \mathrm{min})$ was performed during 4 hours of a hyperinsulinemic clamp involving the infusion of high-dose insulin $(18 \mathrm{mU} / \mathrm{kg} / \mathrm{min})$ and glucose at variable rates to keep animals euglycemic. (c) Intracellular levels of UDPGlcNAc in skeletal muscle of rats infused with saline $(C)$ or glucosamine $(G)$. ${ }^{*} P<0.005$. (d) Western blot analysis of O-GlcNAc modification in skeletal muscle. Extracts from glucosamine-treated animals (G) and saline-infused animals (C) are compared. 
Table 1

Metabolic parameters of the experimental groups before and during the insulin clamp

\begin{tabular}{|c|c|c|c|c|}
\hline \multirow{3}{*}{$n$} & \multicolumn{2}{|c|}{ GlcN } & \multicolumn{2}{|c|}{ Control } \\
\hline & \multicolumn{2}{|c|}{11} & \multicolumn{2}{|c|}{11} \\
\hline & Basal & Clamp & Basal & Clamp \\
\hline Body weight (g) & $316.8 \pm 8.6$ & - & $318.5 \pm 5.4$ & - \\
\hline Glucose (mM) & $7.8 \pm 0.3$ & $7.7 \pm 0.4$ & $7.7 \pm 0.2$ & $7.8 \pm 0.3$ \\
\hline $\mathrm{FFA}(\mathrm{mM})$ & $0.62 \pm 0.06$ & $0.45 \pm 0.05$ & $0.56 \pm 0.04$ & $0.4 \pm 0.1$ \\
\hline Leptin (ng/ml) & $1.32 \pm 0.16$ & $3.3 \pm 0.32^{\mathrm{A}, \mathrm{B}}$ & $1.4 \pm 0.26$ & $2.27 \pm 0.3$ \\
\hline Rat insulin (ng/ml) & $1.35 \pm 0.2$ & - & $1.45 \pm 0.26$ & - \\
\hline Clamp insulin $(\mu \mathrm{U} / \mathrm{ml})$ & - & $504 \pm 59$ & - & $485 \pm 59$ \\
\hline \multicolumn{5}{|l|}{ Overfeeding experiments } \\
\hline \multirow{3}{*}{$n$} & \multicolumn{2}{|c|}{$\mathrm{HF}$} & \multicolumn{2}{|c|}{ Control } \\
\hline & \multicolumn{2}{|c|}{5} & \multicolumn{2}{|c|}{4} \\
\hline & Basal & Clamp & Basal & Clamp \\
\hline Body weight (g) & $324 \pm 10$ & - & $298 \pm 7$ & - \\
\hline Change in body weight (g) & $12 \pm 5^{\mathrm{A}}$ & - & $-16 \pm 4.0$ & - \\
\hline Calorie intake (kcal/d) & $126 \pm 8$ & - & $60 \pm 2$ & - \\
\hline Glucose (mM) & $8.1 \pm 1^{\mathrm{B}}$ & - & $6.8 \pm 0.2$ & - \\
\hline FFA (mM) & $0.6 \pm 0.2$ & - & $0.5 \pm 0.3$ & - \\
\hline Leptin (ng/ml) & $2.8 \pm 0.5^{\mathrm{A}}$ & - & $1.2 \pm 0.2$ & - \\
\hline Rat insulin (ng/ml) & $1.7 \pm 0.4^{\mathrm{A}}$ & - & $0.9 \pm 0.2$ & - \\
\hline Clamp insulin $(\mu \mathrm{U} / \mathrm{ml})$ & - & $35.4 \pm 6$ & - & $34.8 \pm 4.7$ \\
\hline
\end{tabular}

The values during the clamp represent steady-state levels obtained by averaging at least five samples during the experimental period. Data are expressed as average $\pm \mathrm{SEM}$. ${ }^{A} P<0.0001 \mathrm{vs}$. basal, ${ }^{\mathrm{B}} P<0.003$ vs. control, ${ }^{C} P<0.05$ vs. basal. HF, high-fat diet.

calculated as the ratio of $\mathrm{CO}_{2}$ production (liters) to $\mathrm{O}_{2}$ consumption (liters). Energy expenditure was calculated as $\mathrm{EE}=\left(3.815+1.232 \times \mathrm{VCO}_{2} / \mathrm{VO}_{2}\right) \times \mathrm{VO}_{2}$.

All values are presented as the mean \pm SE. Unpaired Student's $t$ test was used for statistical analysis. The study protocol was reviewed and approved by the Institutional Animal Care and Use Committee of the Albert Einstein College of Medicine.

\section{Results}

Glucosamine infusion increased skeletal muscle UDPGlcNAc levels and protein glycosylation by O-GlcNAc. The in vivo protocol was designed to generate a significant increase in carbon flux through HBP in order to examine the impact of this nutrient-sensing pathway on gene transcription. We also wished to simulate the increase in the flux of nutrients into skeletal muscle that occurs during short-term overfeeding, while maintaining carefully controlled hormonal and metabolic conditions. To this end, we infused either glucosamine ( $\mathrm{GlcN})$ or vehicle for 4 hours in conscious rats in the presence of insulin clamp conditions (Figure 1b). The two experimental groups were matched for body weight and endocrine/metabolic parameters (Table 1). This experimental protocol resulted in a significant increase (fourfold) in the skeletal muscle concentration of UDP-GlcNAc, the end product of HBP (Figure 1c). To document that the increased availability of intracellular UDP-GlcNAc resulted in increased protein glycosylation by O-GlcNAc, we performed Western blots of total muscle extracts using antibodies specific for $\mathrm{O}$ GlcNAc-modified serine/threonine residues (21). GlcN infusion increased this type of glycosylation in several muscle proteins (Figure 1d). The latter increase in O-GlcNAc modifications was quite variable depending on the specific band, with increases over vehicle ranging from about twofold to more than tenfold.

Activation of HBP decreases expression of genes for oxidative phosphorylation in skeletal muscle. We used high-density DNA microarrays to characterize the changes in gene expression elicited by the activation of HBP in skeletal muscle. Among 8975 mouse genes and expressed sequence tags present on the chip, 97 transcripts were increased and 169 were decreased by GlcN compared with vehicle. Importantly, GlcN significantly decreased the expression of nuclear-encoded mitochondrial

Table 2

List of nuclear-encoded mitochondrial transcripts downregulated by GlcN infusion

Function

Krebs cycle

Respiratory chain complex I

Respiratory chain complex III

Respiratory chain complex IV

Respiratory chain complex $\mathrm{V}$

Substrate shuttles

Fatty acid oxidation

\section{Gene}

Malate dehydrogenase, mitochondrial

Fold decrease

$\mathrm{NADH}$-ubiquinone oxidoreductase MWFE subunit

2.23

$\mathrm{NADH}$-ubiquinone oxidoreductase B22 subunit

2.1

2.755

Ubiquinol-cytochrome $c$ reductase complex core protein-2 precursor $\quad 5.191$

Cytochrome $c$ oxidase, polypeptide VIB

2.741

2.218

2.312

ATP synthase $\varepsilon$ chain

2.184

2.46

2.47

2.64

Skeletal muscle RNA from GlcN-treated animals and saline-infused control animals was compared. Microarray analysis revealed a reduced expression of a cluster of genes involved in substrate oxidation and oxidative phosphorylation. The fold decrease indicates the average change in hybridization levels obtained from three independent experiments. 
a
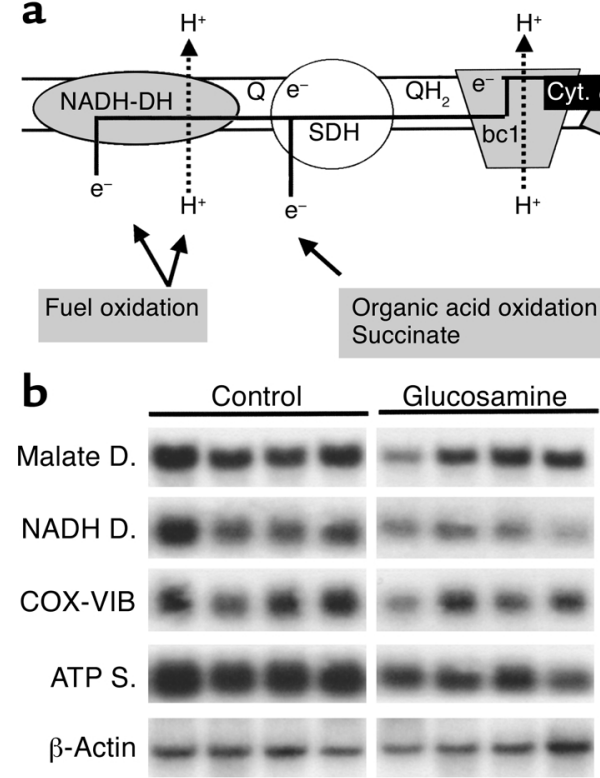

d

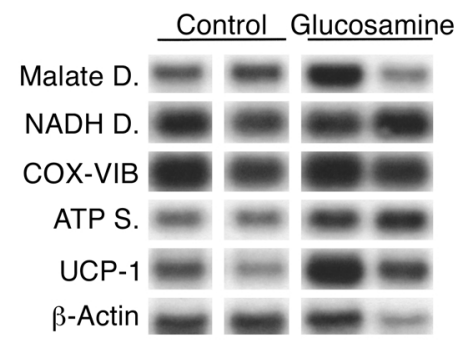

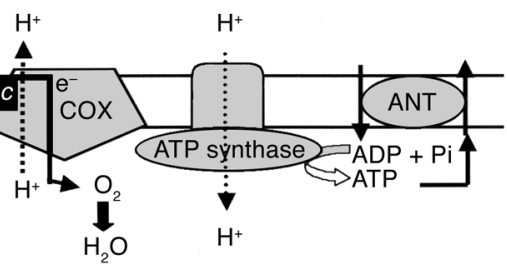

Mitochondrial matrix
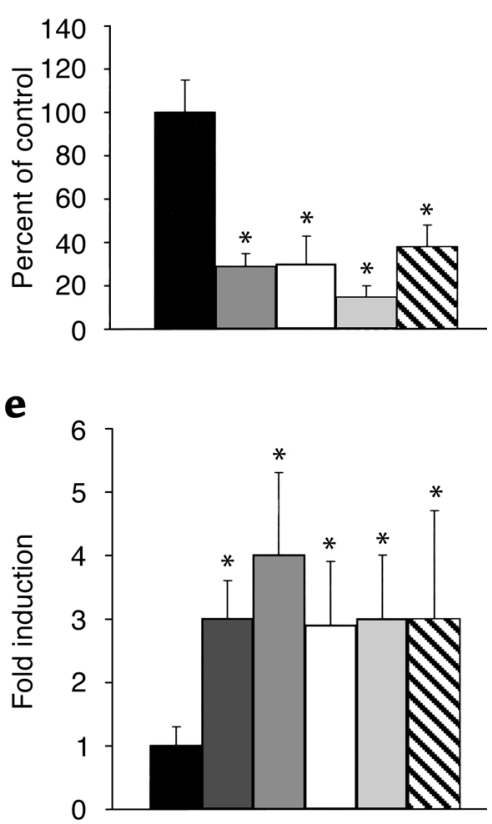

Figure 2

In vivo effect of GlcN on the expression of nuclear-encoded mitochondrial transcripts. (a) Schematic illustration of respiratory chain complexes and oxidative phosphorylation. Electrons $\left(\mathrm{e}^{-}\right)$donated from fuel oxidation are sequentially transferred from ubiquinone $\left(\mathrm{Q}\right.$ and $\left.\mathrm{QH}_{2}\right)$ to cytochrome $c$ (Cyt. c), and finally to oxygen $\left(\mathrm{O}_{2}\right)$. Simultaneously, protons $\left(\mathrm{H}^{+}\right)$are translocated across the inner mitochondrial membrane and create an electrochemical gradient whose energy is eventually used by ATP synthase for ATP formation. An adenine nucleotide translocator (ANT) carries ATP out of the mitochondria in exchange for ADP. The complexes indicated in gray contain one or more subunits downregulated by glucosamine. COX, cytochrome $c$ oxidase; SDH, succinate dehydrogenase. (b) Northern analysis of mitochondrial transcripts in skeletal muscle from glucosamine versus saline infusion (control) with probes encoding mitochondrial malate dehydrogenase, NADH dehydrogenase, cytochrome $c$ oxidase (COX-VIB), ATP synthase, and $\beta$-actin. (c) Quantitation, by densitometry, of Northern blots shown in b. Data are expressed as percent of control hybridization and are normalized with a $\beta$-actin probe. (d) Northern analysis of mitochondrial transcripts in BAT from glucosamine versus saline infusion (control) studies. Probes are the same as in b, except for the BAT-selective UCP-1. (e) Quantitation of Northern blot shown in d. Legend to bar graphs: Black, control; dark gray, UCP-1; medium gray, ATP synthase; white, cytochrome $c$ oxidase VIB; light gray, NADH dehydrogenase; hatched, malate dehydrogenase. ${ }^{*} P<0.01$

proteins involved in oxidative phosphorylation (Table 2). In mitochondria, the reduced NADH produced by the oxidation of nutrients generates energy by donating electrons down an array of carrier molecules called the electron transport chain (Figure 2a). The latter is composed of a series of four respiratory enzyme complexes localized in the mitochondrial inner membrane. The energy released by the electron transport is used to form a proton gradient across the mitochondrial inner membrane, which is in turn used by the ATP synthase complex to generate ATP from ADP and phosphate. A carrier protein (ANT [adenine nucleotide translocator]) exports ATP in exchange for ADP. Oxidative phosphorylation is the major cellular mechanism for the generation of energy in the form of ATP. Activation of HBP decreased the expression of one or more subunits from all but one of the respiratory chain complexes, as shown in Table 2 and Figure 2a. Additionally, GlcN also downregulated the expression of selective genes involved in fatty acid oxidation, mitochondrial substrate shuttles, and Krebs cycle. To validate the results obtained by analysis of microarrays, we next measured the levels of mRNA for selective mitochondrial proteins by Northern blot analysis (Figure 2b). Mitochondrial malate dehydrogenase (Krebs cycle) and the cytochrome $c$ oxidase subunit VIB (respiration chain) were decreased by two- to threefold, while $\mathrm{NADH}$ dehydrogenase and the $\varepsilon$ subunit of ATP synthase (respiration chain) were decreased by threeto fourfold (Figure 2b). Northern analysis of uncoupling protein-3 (UCP-3) mRNA did not reveal any significant change with HBP activation (data not shown).

The effect of HBP on the expression of genes for oxidative phosphorylation is limited to skeletal muscle. Since brown adipose tissue (BAT) plays an important role in the regulation of energy expenditure, we next asked whether the activation of HBP also altered the expression of oxidative phosphorylation genes in BAT. Northern blot analysis performed at the completion of the GlcN infusion revealed that the expression of uncoupling protein-1 (UCP-1), which dissipates energy into heat by uncoupling oxygen consumption from ATP synthesis, and of genes of oxidative phosphorylation was actually increased in BAT. Mitochondrial malate dehydrogenase and cytochrome $c$ oxidase were induced two- to threefold, while NADH dehydrogenase, UCP-1, and the $\varepsilon$ subunit of ATP synthase were increased four- to fivefold (Figure 2, $d$ and e). These findings suggest that increased dissipation of energy in BAT may partly compensate for the marked decrease in mitochondrial respiration in skeletal muscle. 
Activation of HBP in skeletal muscle decreases wholebody oxygen consumption and energy expenditure. If the above transcriptional regulation leads to physiologically relevant changes in mitochondrial function, one should be able to measure alterations in in vivo respiratory exchanges. Thus, we asked whether a prolonged activation of HBP in skeletal muscle decreases whole-body oxygen consumption and energy expenditure. To this end, we measured respiratory exchanges by indirect calorimetry before and after $\mathrm{GlcN}$ infusion. Sprague-Dawley (S-D) male rats were matched for food intake, body weight, and base-line energy expenditure and were randomized to receive either $\mathrm{GlcN}$ or vehicle infusion for 4 hours during insulin clamp studies (Figure 3a). After the completion of the clamp study, all rats were placed back into individual metabolic cages and their movements and gas exchange data were recorded. The post-clamp calorimetry was performed 3 hours before the onset of the light-dark cycle to allow the collection of gas exchanges at rest.

Prior to infusions, oxygen consumption and energy expenditure were similar in the two groups (Figure 3, $\mathrm{b}$ and c). However, activation of HBP by GlcN infusion markedly decreased oxygen consumption by $22 \%$
$(892.8 \pm 26.2 \mathrm{ml} / \mathrm{kg} / \mathrm{h}$ vs. $1150.3 \pm 77.5 \mathrm{ml} / \mathrm{kg} / \mathrm{h}$ of vehicle) and energy expenditure by $33 \%(1.17 \pm 0.05 \mathrm{kcal} / \mathrm{h}$ vs. $1.75 \pm 0.15 \mathrm{kcal} / \mathrm{h}$ of vehicle) compared with either base-line or vehicle (Figure 3, b and c).

Total oxygen consumption and energy expenditure remained significantly lower in the GlcN-infused rats for 4 hours after the end of GlcN infusion compared with vehicle (Figure 3, $d$ and e). The RQ did not change significantly immediately after the clamp studies (Figure 3f). However, during the last 5 hours of the experiment, the RQ of the control group decreased from $0.78 \pm 0.04$ at 1 hour to $0.66 \pm 0.03$ at 17 hours from the end of the clamp study. By contrast, the RQ of the GlcN group did not change significantly $(0.79 \pm 0.02$ at 1 hour vs. $0.77 \pm 0.02$ at 17 hours). This lack of transition to a lower RQ during fasting may reflect the downregulation of genes involved in fatty acid oxidation. Alternatively, HBP may activate net lipid biosynthesis, which would also result in a higher RQ. The effects of $\mathrm{HBP}$ on $\mathrm{O}_{2}$ consumption and energy expenditure could not be ascribed to differences in physical activity during the gas exchange measurements (Figure $3 \mathrm{~g}$ ).

Thus, activation of HBP leads to marked decreases in whole-body energy expenditure, consistent with the
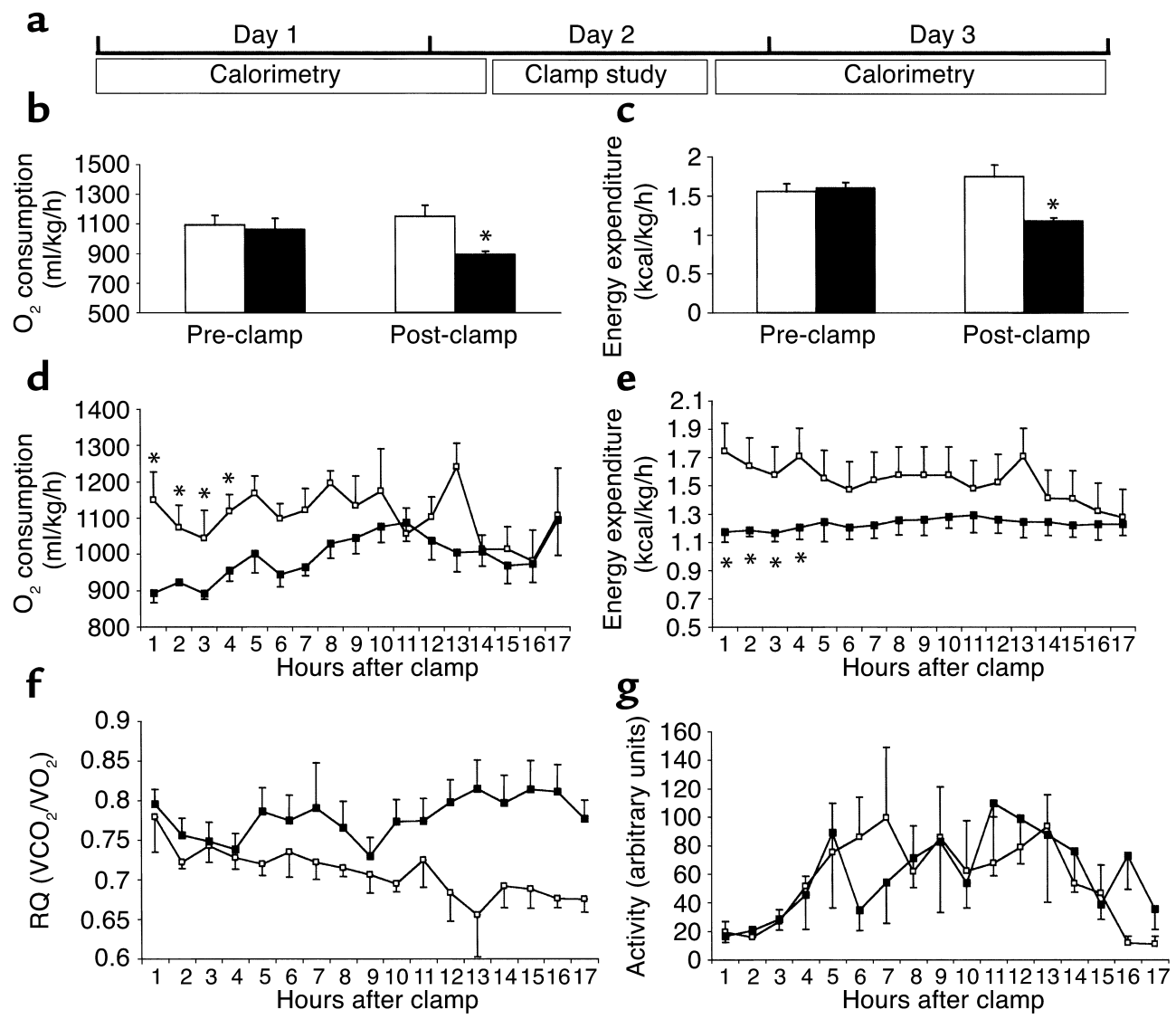

Figure 3

Effect of GlcN on whole-body $\mathrm{O}_{2}$ consumption and energy expenditure. (a) Scheme of experimental design. Indirect calorimetry was performed on rats before and immediately after the completion of clamp studies. (b and c) Resting $\mathrm{VO}_{2}(\mathbf{b})$ and energy expenditure (c) are decreased in $\mathrm{GlcN}$ treated animals (black bars) as compared with saline-injected animals (white bars). (d-g) Time course of $\mathrm{VO}_{2}$ (d), energy expenditure (e), RQ (f), and activity (g), after completion of clamp studies in GlcN-treated (black squares) and saline-injected animals (white squares). ${ }^{*} P<0.05$. 

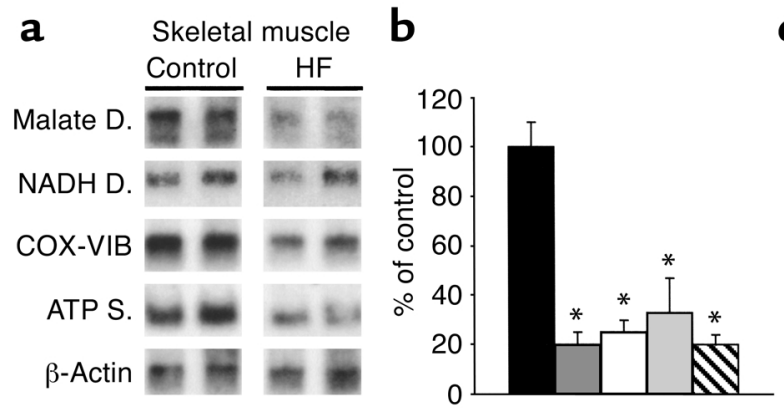

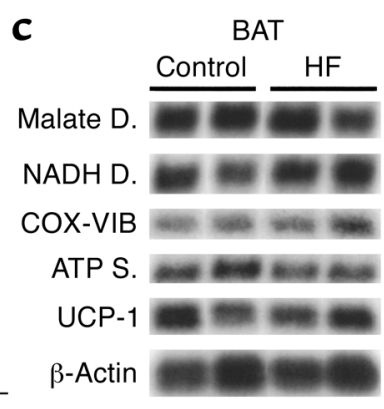

\section{Figure 4}

Effect of short-term overfeeding on the expression of genes of oxidative phosphorylation. (a and b) Northern blot (a) and quantitation (b) in skeletal muscle of animals overeating a high-fat diet (HF) compared with control animals. (c) Northern analysis of genes for oxidative phosphorylation in BAT of overeating rats versus control. Legend to bar graphs: Black, control; medium gray, ATP synthase; white, cytochrome $c$ oxidase VIB; light gray, NADH dehydrogenase; hatched, malate dehydrogenase. ${ }^{*} P<0.001$.

downregulation of genes involved in oxidative phosphorylation and fatty acid oxidation in skeletal muscle.

Overfeeding downregulates the expression of genes for oxidative phosphorylation in skeletal muscle. Activation of HBP rapidly enhances cellular glycosylation of proteins by O-GlcNAc and decreases the expression of key mitochondrial proteins involved in oxidative phosphorylation. In order to evaluate this link between nutrient sensing and energy expenditure in a more physiological setting, we next asked whether short-term voluntary overfeeding also induces a downregulation of genes for oxidative phosphorylation in skeletal muscle. S-D rats were given a palatable high-fat diet for 3 days. This resulted in an approximately twofold increase in caloric intake compared with controls rats who were eating regular chow (Table 1) (18). However, while body weight was markedly increased (Table 1), energy expenditure and oxygen consumption were only modestly higher (by $\sim 7 \%$; data not shown) in response to short-term overfeeding. This nutritional manipulation also resulted in moderate increases in skeletal muscle $(-35 \%)$ and liver $(\sim 60 \%)$ levels of UDPGlcNAc. Using Northern analysis, we next measured the expression of the transcripts downregulated by HBP in muscle (Figure 4a). In this animal model, 3 days of voluntary overfeeding markedly downregulated the expression of genes encoding mitochondrial proteins (Figure 4, $a$ and $b$ ). In particular, overfeeding decreased the skeletal muscle mRNA for mitochondrial malate dehydrogenase and the $\varepsilon$ subunit of ATP synthase four- to fivefold, and the mRNA for NADH dehydrogenase and cytochrome $c$ oxidase VIB subunit two- to threefold. However, shortterm overfeeding failed to alter the expression of these genes and of UCP-1 in BAT (Figure 4c).

\section{Discussion}

Mammals rapidly adapt to changes in caloric intake via compensatory changes in substrate oxidation and energy expenditure $(4,22)$. Thus, energy expenditure increases in response to voluntary overfeeding in an apparent attempt to limit energy "overflow" and storage. On the other hand, there are considerable interindividual variations in the effects of short-term overfeeding on energy expenditure (1). These variations appear to be genetically determined and partly due to variable expression and/or penetrance of thrifty genes, which limit the compensatory increase in energy expenditure in response to nutrient excess $(2,3)$. At the cellular level, long-term ATP production is maintained within a relatively tight range in most tissues, including resting skeletal muscle. It is hypothesized herein that a robust activation of a nutrient-sensing pathway is a signal of energy overflow. This signal in turn reduces the efficiency of ATP production in an attempt to limit marked excursions in the rate of cellular respiration.

Consistent with this hypothesis, here we show that overstimulation of HBP inhibits the expression of nuclear-encoded mitochondrial proteins involved in oxidative phosphorylation and substrate oxidation in skeletal muscle. Most important, this in turn leads to a significant decrease in whole-body oxygen consumption and energy expenditure. However, it should be noted that the changes in energy metabolism in response to activation of HBP are not limited to skeletal muscle. This signal of fuel abundance also resulted in a moderate increase in circulating leptin concentrations and in the induction of UCP-1 and of nuclear-encoded mitochondrial transcripts in BAT. The latter effects of GlcN infusion appear to represent attempts to dispose of the perceived abundance of fuel in skeletal muscle by increasing energy dissipation elsewhere (in BAT) and by activating leptin-driven neuroendocrine responses.

Despite these adaptive responses to HBP activation, whole-body energy expenditure was markedly reduced, suggesting that the inhibition of oxygen consumption in muscle outweighed the changes in mitochondrial bioenergetics in BAT. Thus, we speculate that the effects of HBP activation on energy metabolism are tissue-specific. In skeletal muscle, this signal of nutrient abundance limits oxidative stress by decreasing substrate oxidation and oxygen consumption, while in BAT it attempts to dissipate excess energy via the uncoupling of oxidative phosphorylation. The moderate increase in circulating leptin induced by $\mathrm{GlcN}$ infusions may play a role in the compensatory increase in the expression of UCP-1 (23) and selective mitochondrial proteins in BAT. Alternatively, the molecular mechanism(s) by which HBP modulates the expression of mitochondrial proteins in a tissue-specific manner may in part relate to the control of SP1 activity via O-GlcNAc modification $(13,24,25)$. In fact, UCP1 and the other genes encoding for proteins in the oxidative phosphorylation and substrate oxidation pathways 
affected by HBP contain SP1-binding sites in their promoters (25-28). Interestingly, SP1 can function as both a positive and a negative regulator of transcription $(25,26)$.

If this link between nutrient sensing and mitochondrial function is relevant to physiological states of nutrient excess, it should also operate during voluntary increases in caloric intake. Indeed, in the present study, short-term voluntary overfeeding reproduced the effects of HBP activation on nuclear-encoded mitochondrial genes. Northern analyses indicate that the extent of transcriptional changes in skeletal muscle following 3 days' overfeeding was quite comparable with that obtained with GlcN infusions.

Short-term overfeeding results in variable increases in body weight and fat mass in different individuals and in various strains of laboratory animals (1-5). This variability is partly due to differences in the compensatory increase in energy expenditure normally designed to match the marked increase in calorie intake. For example, the S-D rats in the present study increased their caloric intake approximately twofold, but they only modestly increased their energy expenditure $(\sim 7 \%)$. This defective or delayed response to increased calorie intake is partly responsible for the rapid increase in body weight in this animal model (29). Thus, we hypothesize that the increased activation of HBP is likely to limit the compensatory increase in energy expenditure during voluntary overfeeding.

In line with the thrifty genotype hypothesis, activation of HBP in the presence of enhanced nutrient availability may have conferred a selective survival advantage by favoring the storage of excess nutrients as fat during periods of sporadic food availability. On the other hand, increased caloric intake for 3 days failed to enhance the expression of UCP-1 and other mitochondrial proteins in BAT. This is at odds with the effects of short-term activation of HBP, which significantly induced these genes in BAT. This may be due to the short duration of overfeeding in the present experiments. Alternatively, it is conceivable that rapid onset of leptin resistance may partly account for the lack of compensatory activation of UCP- 1 and genes for oxidative phosphorylation in BAT. In fact, we recently reported that voluntary hyperphagia in obesity-prone rats rapidly induced insulin and leptin resistance (18). The latter was characterized by a marked decrease in leptin's ability to modulate both liver metabolism and food intake.

Thus, in keeping with Neel's thrifty genotype hypothesis (6), a sustained increase in the availability of nutrients may trigger biological actions designed to increase the efficiency of energy storage by limiting fuel oxidation and ATP production. This biochemical link between nutrient excess and energy expenditure may play a significant role in the pathophysiology of obesity.

\section{Acknowledgments}

This work was supported by grants from the National Institutes of Health (DK 48321 and DK 45024 to L. Rossetti), from the AECOM Diabetes Research \& Training Center (DK 20541). S. Obici was the recipient of a post-doctoral fellowship and a Junior Faculty Award from the American Diabetes Association.

1. Levine, J.A., Eberhardt, N.L., and Jensen, M.D. 1999. Role of nonexercise activity thermogenesis in resistance to fat gain in humans. Science. 283:212-214.

2. Bouchard, C., et al. 1990. The response to long-term overfeeding in identical twins. N. Engl. J. Med. 322:1477-1482.

3. Ravussin, E., et al. 1988. Reduced rate of energy expenditure as a risk factor for body-weight gain. N. Engl. J. Med. 318:467-472.

4. Leibel, R.L., Rosenbaum, M., and Hirsch, J. 1995. Changes in energy expenditure resulting from altered body weight. N. Engl. J. Med. 332:621-628.

5. Schwartz, M.W., Woods, S.C., Porte, D., Jr., Seeley, R.J., and Baskin, D.G. 2000. Central nervous system control of food intake. Nature. 404:661-671.

6. Neel, J.V. 1999. The "thrifty genotype" in 1998. Nutr. Rev. 57(Suppl. II):S2-S9.

7. Marshall, S., Bacote, V., and Traxinger, R. 1991. Discovery of a metabolic pathway mediating desensitization of the glucose transport system: role of hexosamine biosynthesis in the induction of insulin resistance. J. Biol. Chem. 266:4706-4712.

8. McClain, D.A., Alexander, T., Cooksey, R.C., and Considine, R.V. 2000. Hexosamines stimulate leptin production in transgenic mice. Endocrinology. 141:1999-2002.

9. Wells, L., Vosseller, K., and Hart, G.W. 2001. Glycosylation of nucleocytoplasmic proteins: signal transduction and O-GlcNAc. Science. 291:2376-2378.

10. Sayeski, P.P., and Kudlow, J.E. 1996. Glucose metabolism to glucosamine is necessary for glucose stimulation of transforming growth factor-alpha gene transcription. J. Biol. Chem. 271:15237-15243.

11. Cheng, X., and Hart, G.W. 2001. Alternative O-glycosylation/O-phosphorylation of serine- 16 in murine estrogen receptor beta: post-translational regulation of turnover and transactivation activity. J. Biol. Chem. 276:10570-10575.

12. Hanover,J.A., Cohen, C.K., Willingham, M.C., and Park, M.K. 1987. O-linked $\mathrm{N}$-acetylglucosamine is attached to proteins of the nuclear pore. Evidence for cytoplasmic and nucleoplasmic glycoproteins. J. Biol. Chem. 262:9887-9894.

13. Han, I., and Kudlow, J.E. 1997. Reduced O glycosylation of Sp1 is associated with increased proteasome susceptibility. Mol. Cell. Biol. 17:2550-2558.

14. Rossetti, L., Hawkins, M., Chen, W., Gindi, J., and Barzilai, N. 1995. In vivo glucosamine infusion induces insulin resistance in normoglycemic but not in hyperglycemic conscious rats. J. Clin. Invest. 96:132-140.

15. Veerababu, G., et al. 2000. Overexpression of glutamine: fructose-6-phosphate amidotransferase in the liver of transgenic mice results in enhanced glycogen storage, hyperlipidemia, obesity, and impaired glucose tolerance. Diabetes. 49:2070-2078.

16. Wang, J., Liu, R., Hawkins, M., Barzilai, N., and Rossetti, L. 1998. A nutrientsensing pathway regulates leptin gene expression in muscle and fat. Nature. 393:684-688.

17. Wang, J., et al. 1999. The effects of leptin on Lep expression is tissue-specific and nutritionally regulated. Nat. Med. 5:895-899.

18. Wang, J., et al. 2001. Overfeeding rapidly induces leptin and insulin resistance. Diabetes. 50:2786-2791.

19. Cheung, V.G., et al. 1999. Making and reading microarray. Nat. Genet. 21:15-19.

20. Eisen, M.B., and Brown, P.O. 1999. DNA arrays for analysis of gene expression. Methods Enzymol. 303:179-205.

21. Comer, F.I., Vosseller, K., Wells, L., Accavitti, M.A., and Hart, G.W. 2001. Characterization of a mouse monoclonal antibody specific for O-linked N-acetylglucosamine. Anal. Biochem. 293:169-177.

22. Lowell, B.B., and Spiegelman, B.M. 2000. Towards a molecular understanding of adaptive thermogenesis. Nature. 404:652-660.

23. Cusin, I., et al. 1999. Chronic central leptin infusion enhances insulin-stimulated glucose metabolism and favors the expression of uncoupling proteins. Diabetes. 47:1014-1019.

24. Du, X.L., et al. 2000. Hyperglycemia-induced mitochondrial superoxide overproduction activates the hexosamine pathway and induces plasminogen activator inhibitor-1 expression by increasing Sp1 glycosylation. Proc. Natl. Acad. Sci. USA. 97:12222-12226.

25. Yang, X., et al. 2001. O-linkage of $\mathrm{N}$-acetylglucosamine to Sp1 activation domain inhibits its transcriptional capability. Proc. Natl. Acad. Sci. USA. 12:6611-6616.

26. Zaid, A., et al. 1999. On the role of the general transcription factor Sp1 in the activation and repression of diverse mammalian oxidative phosphorylation genes. J. Bioenerg. Biomembr. 31:129-135.

27. Zaid, A., Hodny, Z., Li, R., and Nelson, B.D. 2001. Sp1 acts as a repressor of the human adenine nucleotide translocase-2 (ANT2) promoter. Eur. J. Biochem. 268:5497-5503.

28. Scarpulla, R.C. 1997. Nuclear control of respiratory chain expression in mammalian cells. J. Bioenerg. Biomembr. 2:109-119.

29. Rothwell, N.J., and Stock, M.J. 1979. A role for brown adipose tissue in dietinduced thermogenesis. Nature. 281:31-35. 